\title{
Assessing Phonological Awareness in Kindergarten Children: Issues of Task Comparability
}

\author{
Keith E. Stanovich \\ Oakland University \\ Anne E. Cunningham \\ University of Michigan \\ AND \\ Barbara B. Cramer \\ Oakland University
}

\begin{abstract}
Ten different phonological awareness tasks were administered to a group of kindergarten children whose reading ability was assessed 1 year later. The extraneous cognitive requirements inherent in the tasks varied widely. The children's performance on three tasks that involved a rhyming response was at ceiling, and these tasks did not correlate with subsequent reading progress. The other seven measures were all moderately related to later reading ability and, employed in sets, were very strong predictors. The relative predictive accuracy of the phonological tasks was equal to or better than more global measures of cognitive skills such as an intelligence test and a reading readiness test. The phonological tasks had a large amount of common variance. Factor analysis revealed only one factor on which all the nonrhyming phonological tasks loaded highly. The results bolster the construct validity of phonological awareness, indicate considerable comparability and interchangeability among the tasks used to measure the construct, and are encouraging as regards the possible use of such tasks in predictive test batteries.
\end{abstract}

\section{Researchers interested in the cognitive determinants of early reading acquisition have increasingly focused on phonological awareness as a}

The authors thank Cecilia Wiar, Principal, and the teachers and students of Clarkston Elementary School, Clarkston, MI, for their cooperation. Anita Davison and Maryanne Dedrick deserve special thanks for their enthusiatic participation. The authors thank Dorothy Feeman and Ruth Nathan for their comments of the manuscript. Requests for reprints should be sent to Keith E. Stanovich, Department of Psychology, Oakland University, Rochester, MI 48063. 
potentially important variable. There is now a substantial body of evidence indicating that tasks that in some way tap phonological awareness are moderate to strong predictors of the speed with which children acquire reading fluency in the early grades (Bradley \& Bryant, 1978; Calfee, Lindamood, \& Lindamood, 1973; Fox \& Routh, 1976, 1980, 1983; Golinkoff, 1978; Helfgott, 1976; Jorm \& Share, 1983; Liberman, 1973, 1982; Rozin \& Gleitman, 1977; Treiman \& Baron, 1981; Williams, 1980). The interest in this particular cognitive skill has been fueled by recent evidence indicating that the early development of phonological awareness is causally linked to rapid reading acquisition (Bradley \& Bryant, 1983; Perfetti, Beck, \& Hughes, 1981; Treiman \& Baron, 1983; Williams, 1980).

A large number of different experimental paradigms have been used to assess phonological awareness, including rhyming tasks, phoneme segmentation tasks, matching tasks, phoneme substitution tasks, blending tasks, and phoneme counting tasks, to name just a few (see Lewkowicz, 1980 , for a useful typology). The plethora of tasks, however, has made a consolidation of the knowledge gained from studies in this area very difficult. All of the tasks that have been used involve many cognitive processes (e.g., short-term memory, stimulus comparison, processing of task instructions) in addition to the phonological analysis ability that is the focus of interest. Without careful task analysis and comparison it will remain unclear to what extent the predictive power of these tasks resides in the phonological ability or the other extraneous cognitive processes.

The present authors are not the first to recognize that a lack of direct task comparisons is the current Achilles' heel of the phonological awareness literature. After a thorough review of the existing research Lewkowicz (1980) observed that

There has been surprisingly little comparison, at least in print, of one phonemic awareness task with another. There has been little analysis of similarities and differences between tasks, of relative difficulty of tasks or of which tasks are most closely related to the reading process and are most likely to facilitate learning to read. In my opinion, this lack of in-depth analysis of phonemic awareness tasks and their relationship to reading has resulted in the obscuring of some important differences between the tasks, and, as a consequence, in the failure of researchers to focus on the most important tasks and questions that need to be asked about them. (pp. 686-687)

Three years later the situation remained much the same, and Backman (1983) concluded from her results that

Tasks which on the surface appear to be measuring the same phenomenon may in fact require different degrees of linguistic awareness, or may differ in their cognitive requirements. . . . We must not talk about phoneme segmentation per se in relation to reading, but segmentation within the context of a particular task. 
... Obviously, ease of understanding task requirements is intimately related to the phenomenon of "linguistic insight" we are interested in. (pp. 476-477)

Both Lewkowicz (1980) and Backman (1983) emphasized that differing cognitive requirements could lead to a divergence in the results obtained from different tasks. However, it is equally true that similar extraneous cognitive requirements could lead to a convergence of results from tasks that actually tap different aspects of phonological awareness (or that tap the same aspect to differing degrees). Actually, the literature on phonological awareness has shown considerable convergence despite the plethora of paradigms that have been used and the absence of extensive direct task comparisons. The fact that a wide variety of tasks has converged on similar conclusions in this area of research is encouraging. However, the general absence of direct comparisons between tasks in the published literature places arguments for convergence on shaky ground. Suspicion will always remain that the convergence is spurious, the result of the other cognitive and linguistic requirements of the phonological tasks. Thus, it is essential that some attempt be made to directly assess the relationships between phonological tasks and determine their degree of convergence. Otherwise, the impact of the strong results previously obtained in this research area may be muted due to lingering doubts about construct validity. Also, as Lewkowicz (1980) noted, multivariate studies of phonological awareness tasks would necessarily address another important question, that of potential task differences in predictive accuracy.

The present study attempted to address these issues. Ten different phonological tasks were administered to a group of kindergarten subjects. The tasks were of several different types, covering many of the categories (e.g., word-to-word matching, rhyme recognition, phoneme deletion, phoneme substitution, and identification of missing phoneme) included in the classification system developed by Lewkowicz (1980). Some tasks required abstraction of the initial phoneme, while others focused on the final phoneme. Finally, the critical linguistic construction that was used in the instructions to the child varied across tasks (e.g., "same," "different," "not same"). In short, the phonological task domain was widely sampled in terms of task type, location of phonological contrast, and task instructions. Whether the differing cognitive requirements of the tasks are reflected in the patterns of the performance relationships should be revealed by correlational analyses. Conversely, the same correlational analyses will give a rough indication of the degree to which these tasks tap a similar underlying construct of phonological awareness. It should be possible to detect performance convergences that are simply due to similarities in extraneous task requirements, because the latter varied between tasks to differing degrees.

The important issue of the relative predictive power of different pho- 
nological awareness tasks was addressed by obtaining a standardized measure of reading ability on the same subjects at the end of first grade. Thus, correlations between the performance on the phonological measures in kindergarten and reading achievement at the end of first grade could be assessed.

As more research attention focuses on the theoretical importance of measures of phonological awareness, practitioners will naturally question whether the relationships between these tasks and reading ability have any degree of practical utility. For example, the question of how these measures relate to other more global prereading assessment devices such as readiness tests and intelligence tests, and how all of these measures compare in predictive power will be raised. These questions were also addressed in the present study by administering a standardized reading readiness test and a standardized general intelligence test to the sample of kindergarten subjects.

\section{METHODS}

\section{Subjects}

Fifty-eight subjects ( 29 males and 29 females) were recruited from three kindergarten classrooms in a predominantly middle-class elementary school. Nine subjects ( 4 males and 5 females) failed to follow the instructions on several tasks, leaving a total of 49 subjects for subsequent analysis. For example, several of these subjects scored zero on the substitute initial consonant and rhyme supply tasks because they gave random responses or semantic associates rather than rhymes. These subjects completed some of the other more difficult tasks, suggesting that their failure on the rhyme supply was due to a failure to understand the instructions. The status of these subjects as multivariate outliers was confirmed by using the objective methods described in Tabachnick and Fidell (1983). The mean age of the 49 subjects was 6 years and 2 months ( $S D=4.4$ months) at the time of testing. The children were administered a battery of 10 phonological tasks in May of the school year by the same experimenter. The prereading sections of the Metropolitan Readiness Tests (Level 2, Form P) were administered to all of the subjects. The mean prereading skills composite score was $47.9(S D=13.8$; mean percentile rank $=47 \%$ ). Forty-six of the forty-nine children were also administered the Otis-Lennon School Ability Test (Primary 1, Form R). The mean score on the Otis-Lennon was $37.8(S D=10.1)$, while the mean School Ability Index (IQ) was $103.8(S D=16.4)$. The two standardized measures were administered in late May and early June, following administration of the 10 phonological tasks. Thirty-one of the subjects (16 males and 15 females) were available for testing the following year. In May these children were administered the Reading Survey Test (Form 
JS, Primary Level 1) of the Metropolitan Achievement Tests. The mean raw score on this test was $42.3(S D=12.4)$ and the mean grade equivalent was $3.1(S D=1.6)$.

\section{Tasks and Procedure}

Ten phonological awareness tasks were individually administered to the subjects. Each task took approximately $10 \mathrm{~min}$. to complete and was administered on a separate day. The order of presentation was rhyme supply, rhyme choice, initial consonant same, final consonant same, strip initial consonant, substitute initial consonant, initial consonant not same, final consonant different, initial consonant different, and supply initial consonant. The subject's score on each task was the total number of correct responses, and the maximum score on each was 10 . In tasks involving a multiple choice the position of the correct alternative was randomly determined and occurred with approximately equal frequency in all positions. The 10 experimental trials for each task were preceded by 3-5 practice trials during which the experimenter ensured that the child understood the task. On these trials the experimenter gave feedback on the correctness of the subject's response. In the case of an incorrect response the subjects were told the correct answer and why it was correct. Following the practice trials the subjects were told that the experimenter could no longer help them and that they were simply to try their best. Subjects were also always told that if they did not know the answer they were to guess.

Rhyme supply. This task assessed children's ability to provide a word that rhymed with the target word. The 10 experimental words were nose, pup, sky, toy, hill, wing, mouse, tip, note, and look. The words were orally presented to the subjects. The subjects were told the experimenter would say a word aloud to them and that their task was to provide another word that rhymed or had the same ending sound as the target word. For practice, the experimenter instructed the subject to listen to the words fish-dish. The subjects were then told to say these words out loud. The subjects were told that both words ended with the -ish sound (that is, that they rhymed). Following the practice trials the experimenter pronounced the 10 experimental words aloud to the subjects, and the subjects responded orally.

Rhyme choice. In this task subjects were provided with the stimulus word and asked to choose 1 of 3 words that rhymed with it. The 10 experimental words were star, mop, green, plane, clown, flash, cake, jump, box, and jeep. The subjects were told to listen closely to the target word and the following 3 words. Their task was to choose a word that rhymed with the target word. The experimenter began with two explicit examples: "Listen to the word pet. Now saw the word pet. Tell me which of these three words rhymes with pet: barn, net, hand." The 
experimenter then explained to the child how he was correct or incorrect and the reasons why. Following the remaining practice trials, the 10 experimental trials were administered.

Initial consonant same. This task consisted of 3 practice and 10 experimental multiple choice trials. The 10 experimental words were milk, pear, fan, bone, soap, tent, leg, duck, nest, and key. In the practice trials, subjects were instructed to listen closely to the beginning sound of the target word. The subjects were then instructed to say the target word aloud. The experimenter then pronounced the target word followed by 3 words. Their task was to decide which had the same initial sound as the target word.

Final consonant same. This task consisted of 3 practice and 10 experimental multiple choice trials. Each trial had a target word followed by 3 alternatives. The 10 experimental target words were worm, cup, pan, beat, leaf, bud, house, hook, nail, and bug. The subjects were instructed to listen closely to the target word and the 3 following words. Their task was to choose the word that shared the same ending sound as the target. A picture of the target word was provided for the subject. The picture remained in front of the subject during the trial. It was hypothesized that providing a picture of the target word would reduce memory load for the subject. This procedure, however, was followed only for this task. The experimenter provided the following example to the subject, "Say the word meat aloud and listen to the ending sound. If I say the word meat and then fin, coat, glass, which word would you say has the same ending sound as meat?" A picture of the target word was placed on the table in front of the subject when the experimenter said the word "meat." The experimenter then explained to the subject why the response was correct or incorrect and the remaining practice trials and the 10 experimental trials were completed.

Strip initial consonant. In this task subjects were required to delete the initial phoneme of a word and pronounce the embedded word that remained. This task was originally used by Bruce (1964) and Calfee, Chapman, and Venezky (1972) and adapted for this experiment. The 10 experimental words were pink, told, man, nice, win, bus, pitch, car, hit, and pout. The experimenter instructed the subjects to listen closely to the target word and then remove the first sound. The experimenter provided the example, "Listen to the word task. If you take away the / $t /$ sound, what word is left?" The subject was then told if he was correct or incorrect and why. After the remaining practice trials the 10 experimental trials were administered.

Substitute initial consonant. This task required subjects to isolate the initial sound of a word and then substitute a different sound to produce a new word. The 10 target words were top, bell, lip, fed, gum, sick, pin, cat, sap, and cut. The subjects were told that the experimenter was going 
to say a word, and that their task was to change the word by substituting the initial sound thereby creating a different word. An example was provided by the experimenter, "If I say the word go, and then change the first sound by changing it to $/ \mathrm{n} /$ the new word will be no." The experimenter then asked the subject to try to change the initial phoneme in the word hang. The subjects were told if they were correct or not and the reasons why. Following this example and the remaining practice trials the 10 experimental trials were administered.

Initial consonant different. The subjects were told that the experimenter would read four words out loud to them. Their task was to listen closely to the beginning sound of each word and choose the word that had beginning sound that was different from the other words. The experimenter instructed, "Say the words bag, nine, beach, and bike. Can you tell me which of these words has a different beginning sound-bag, nine, beach, bike?"' Following additional practice trials, the subjects were administered the 10 experimental trials. The 10 correct words were ear, pop, hill, band, arm, give, van, cart, rice, and teeth.

Initial consonant not same. This task is structurally similar to the initial consonant different task except that the directions are phrased in a negative manner. The 10 experimental target words were boy, doll, sun, kite, man, nest, fish, train, pie, and lamp. The subjects were instructed to listen closely to the initial sound of the first word. The experimenter said, "I am going to say a word aloud followed by three more words. Your task is to tell me which word does not begin with the same sound as the first word. Say the word mud. Now say the words mice, dig, and mouth. Can you tell me which word did not have the same beginning sound as mud?" Each subject was told if their answer was correct or incorrect and the reasons why. Following the remaining practice trials the 10 experimental trials were administered.

Final consonant different. In this multiple choice task, subjects were asked to identify one of four words which had a final sound that was different from the others. Three practice and ten experimental trials were administered. The three practice words were rat, can, and log. The 10 experimental target words were ham, cup, leaf, flag, dress, wrist, ball, sand, rain, and desk. The subjects were advised to listen closely to the four words the experimenter would read to them. Their task was to choose the word that had a different ending sound. For example, the experimenter said "Listen to the following four words: rat, dir.e, boat, mitt. Say these words out loud. One of them has a different ending sound. Can you tell me which word has a different sound at the end of the word?" The experimenter then told the subjects when they were correct or incorrect and the reasons why. Following the three practice trials the subjects were informed that the experimenter could no longer 
help them and that if they could not choose the correct word that they should give their best guess.

Supply initial consonant. This task assessed the child's ability to isolate and produce the initial phoneme of a word. Subjects were orally presented a pair of words that were identical except that the initial phoneme had been deleted from the second word. The 10 experimental word pairs were meal-eel, fill-ill, sit-it, land-and, near-ear, pair-air, bend-end, task-ask, date-ate, and can't-ant. The subjects were told that they would be hearing two words that were the same except for the beginning sound. For example, the experimeter said "Say the word cat. Now say $a t$. What sound do you hear in cat, that is missing from at?" The subjects were told the correct answer if they were unable to respond correctly. Following the remaining practice trials the 10 experimental trials were administered.

\section{RESULTS}

Descriptive statistics on each of the 10 phonological awareness measures are presented in Table 1. Hotelling's $T$, calculated with the 10 phonological tasks as the set of dependent measures, indicated no sex differences in the data $(T=8.614, F<1)$, so this variable will not be considered further. A perusal of the means reveals several patterns. The strip initial consonant task was the most difficult task. The mean score on this task was extremely low and the scores were positively skewed. The mode was zero (a score attained by 25 children), but 10 children attained a score of 8 or above. Thus, there is some indication of a bimodal distribution of responses, a pattern that has been observed before with the strip consonant task (see Calfee et al., 1972). Clearly this task exceeds the cognitive and phonological analysis capabilities of many kindergarten children. Nevertheless, the task was a moderately good predictor of firstgrade reading and did correlate with other tasks that had more symmetrical distributions of responses (see below). The supply initial consonant task and both measures involving the final consonant were relatively difficult tasks (although note that the means are not directly comparable because the multiple choice nature of the latter two tasks probably restricts guessing responses).

Tasks where the critical sound contrast was at the beginning of the word were easier than those where the critical sound was at the end of the word. There was some statistical support for this trend. The mean in the initial consonant same task was higher than the mean in the final consonant same task, $t(48)=5.88, p<.001$, and the mean in the initial consonant different task was significantly higher than the mean in the final consonant different task, $t(48)=3.57, p<.01$. Marsh and Mineo (1977) also found that phoneme isolation performance was superior when the critical phonemic contrast was in the initial position, but this pattern 
PHONOLOGICAL AWARENESS

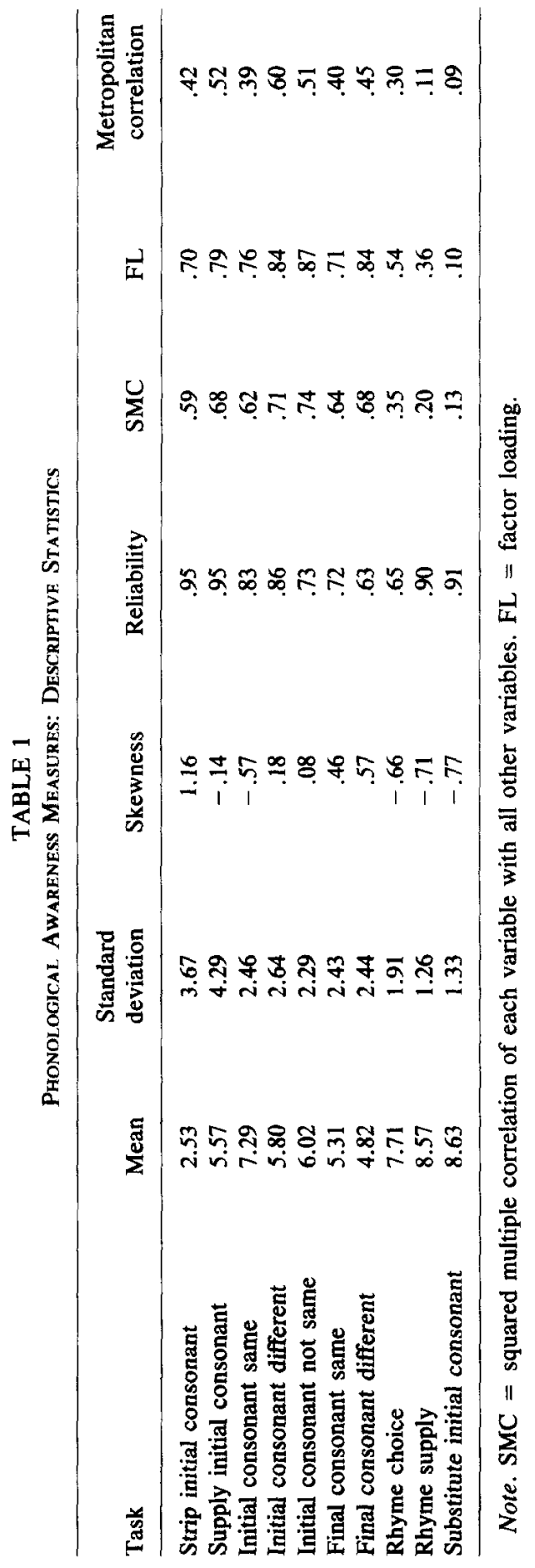


interacted with phoneme class (continuants versus stops). A post hoc analysis of our data revealed no such interaction, error rates being very similar for continuants and stops in all tasks.

The three easiest tasks were substitute initial consonant, rhyme supply, and rhyme choice. Note that the substitute initial consonant task essentially requires the subject to produce a rhyme. It differs from the other two tasks only in the phrasing of the instructions, which do not explicitly mention rhyming. The superior performance on the three rhyming tasks confirms previous research, and the reports of teachers that children often enter school with rhyming skills and that it is the easiest auditory analysis task to teach (see Jusczyk, 1977; Lewkowicz, 1980). Indeed, there is strong evidence that performance on these threc tasks was at ceiling. The rhyming tasks had the three smallest standard deviations and the distributions of all three were characterized by negative skewness. The distribution of responses on the substitute initial consonant task illustrates the ceiling effect. The modal score was 10 (achieved by 16 of the subjects), and the next most frequent score was 9 (achieved by 14 subjects). The ceiling effects apparent in the three rhyming tasks probably account for the fact that these tasks were poor predictors of later reading achievement and were only weakly related to the other phonological awareness measures (see below). Essentially, they suffer from extremely restricted ranges, as indicated by the standard deviations in Table 1, and thus will necessarily fail to correlate with other variables.

The split-half reliability (Spearman-Brown corrected) of each task is also presented in Table 1. In general, the reliabilities of the 10 tasks ranged from low moderate to high. The mean task reliability was .81 . Of course, the magnitudes of the correlations displayed by a given task are limited by the reliability of the task, and this fact should be remembered when interpreting the obtained correlations. In general, however, a consideration of the reliabilities does not attenuate the conclusions drawn here, and in many cases consideration of the reliabilities serves to strengthen the apparent trends. For example, some tasks that were good predictors and that were strongly related to other tasks were only moderately reliable (initial consonant not same), while others that displayed weak relationships were highly reliable (rhyme supply and substitute initial consonant, although the high reliability may also be due to the ceiling effects in the performance on these tasks).

The interrelationships among the phonological awareness tasks are displayed in Table 2 which is a correlation matrix of the ten measures. All correlations larger than .28 are significant at the .05 level. The major data pattern is apparent from a visual inspection of the matrix. The seven nonrhyming tasks show moderate to strong relationships with each other. The substitute initial consonant task does not correlate with anything. The rhyme choice and rhyme supply tasks show weak correlations with 
TABLE 2

INTERCORRELATIONS OF ALL TASKS

\begin{tabular}{lccccccccr}
\hline Task & 2 & 3 & 4 & 5 & 6 & 7 & 8 & 9 & 10 \\
\hline 1. Strip initial consonant & .49 & .45 & .57 & .62 & .66 & .66 & .30 & .18 & -.02 \\
2. Supply initial consonant & & .62 & .68 & .73 & .41 & .69 & .47 & .35 & .01 \\
3. Initial consonant same & & & .62 & .74 & .53 & .60 & .48 & .20 & .09 \\
4. Initial consonant different & & & & .70 & .68 & .72 & .40 & .28 & .17 \\
5. Initial consonant not same & & & & .55 & .71 & .44 & .34 & .11 \\
6. Final consonant same & & & & & & .61 & .39 & .24 & .06 \\
7. Final consonant different & & & & & & .40 & .26 & -.02 \\
8. Rhyme choice & & & & & & & .32 & .16 \\
9. Rhyme supply & & & & & & & & .13 \\
10. Substitute initial consonant & & & & & & & & \\
\hline
\end{tabular}

the other variables. This pattern is quantified in Table 1, which displays the squared multiple correlations of each variable as a dependent variable regressed on the other nine. The values for the seven nonrhyming tasks were very high, approaching their reliabilities in some cases. The seven nonrhyming tasks appear to have much common variance. In contrast, the SMCs of the rhyming tasks were very low (considering the number of predictor variables), ranging from a high of .35 for rhyme choice to a low of .13 for substitute initial consonant. Basically, the extremely restricted range of the scores on the rhyming tasks prevents them from correlating with anything.

The uniformly moderate to high correlations among the seven nonrhyming tasks was somewhat surprising given their different cognitive requirements and moderate reliabilities in some cases. The mean correlation between these seven variables was .62, which is quite high considering that their mean reliability is .81. Also surprising was the relative lack of clusters among the correlations of the seven nonrhyming tasks. The variables were relatively uniformly intercorrelated. These visual impressions from Table 2 were confirmed by a principle factor analysis carried out on the data of all ten tasks. With squared multiple correlations serving as initial commonality estimates, only the first factor exceeded the eigenvalue $>1$ criterion (the eigenvalue of the second factor was .398). This factor accounted for $47.8 \%$ of the total variance in the variables. A stable solution was reached after four iterations, and the factor loadings from this solution are displayed in Table 1. Predictably from the pattern of correlations in Table 2 , the seven nonrhyming tasks had high loadings on the first principle factor, the rhyme choice task had a moderate loading, and the other two rhyming tasks had low loadings.

The last column of Table 1 displays the correlation between each of the phonological measures and the score on the Reading Survey Test of the Metropolitan Achievement Tests that was administered at the end 
of first grade. Correlations larger than .35 are significant at the .05 level. All seven of the nonrhyming tasks displayed significant correlations of moderate strength. Some idea of the relative magnitude of the difference in performance on the phonological tasks between readers of different abilities is provided by Table 3 . There the 31 subjects who were assessed in the first grade have been split into a group of 16 less-skilled readers and a group of 15 skilled readers based on their score on the Reading Survey Test of the Metropolitan. The scores of the two groups were significantly different at the .001 level. The mean grade equivalent of the less-skilled group was 1.9 and the mean grade equivalent of the skilled group was 4.3. From Table 3 it is apparent that all of the measures except two of the rhyming tasks displayed significant differences between the two groups. It is interesting that the skilled group is near ceiling even on some of the nonrhyming tasks, whereas the performance of the lessskilled group is far from ceiling on any of these tasks. The median split thus gives some indication of the large performance disparity that is present on some of the nonrhyming tasks.

In attempting to assess how to best characterize the ability of the phonological tasks to predict first-grade reading levels, it is useful for comparative purposes to consider the results involving the Metropolitan Readiness Tests and Otis-Lennon IQ, two omnibus measures of cognitive skills that were also administered to these children in kindergarten. The prereading readiness test correlated .52 with first-grade reading ability and the correlation between the raw score on the Otis-Lennon and reading ability was .25 . Thus, three of the seven nonrhyming phonological measures (each containing 10 items and taking less than 10 min to administer) predicted first-grade reading ability as well as a standardized readiness test containing six different subsections, and all of the nonrhyming phonological measures were superior to an omnibus IQ test. The mean

TABLE 3

Mean Sources on the Phonological Tasks as a Function of Reader Skill

\begin{tabular}{lccc}
\hline Task & Skilled & Less skilled & $t$ \\
\hline Strip initial consonant & 4.73 & 1.13 & $2.80^{*}$ \\
Supply initial consonant & 9.27 & 4.69 & $3.81^{*}$ \\
Initial consonant same & 9.20 & 7.00 & $3.43^{*}$ \\
Initial consonant different & 8.47 & 4.75 & $5.78^{*}$ \\
Initial consonant not same & 8.07 & 5.56 & $4.52^{*}$ \\
Final consonant same & 7.00 & 4.31 & $3.62^{*}$ \\
Final consonant different & 6.93 & 4.06 & $4.44^{*}$ \\
Rhyme choice & 9.07 & 7.44 & $2.82^{*}$ \\
Rhyme supply & 9.00 & 8.75 & 0.57 \\
Substitute initial consonant & 8.87 & 8.38 & 0.92 \\
\hline
\end{tabular}

$* p<.01$. 
correlation between performance on the seven nonrhyming phonological tasks and performance on the Metropolitan prereading readiness section was .68. The analogous mean correlation with the Otis-Lennon score was .55 .

The trends already described were confirmed in a somewhat different way by regression analyses. A stepwise regession analysis with firstgrade Metropolitan score as the dependent variable and the 10 phonological variables as predictors confirmed the essential redundancy and large variance overlap in the phonological measures. After initial consonant different and initial consonant not same were entered into the equation no other variable made a significant contribution to explaining reading variance. Together, these two variables accounted for $66.2 \%$ of the variance in reading ability (adjusted $R^{2}=.638$ ). Both regression coefficients were significant in the final analysis. The relatively high proportion of variance explained was not primarily due to the optimization criteria of the stepwise regression. The median proportion of variance explained by the 21 different pairs of nonrhyming phonological tasks was $57.6 \%$. This figure is comparable to the $59.1 \%$ of the variance in first-grade reading ability that is accounted for when both the Otis-Lennon and Metropolitan readiness test were employed as predictors. An additional hierarchical regression analysis indicated that after the two phonological measures had been entered into the regression equation the two standardized measures, when entered as a set, did not account for a significant additional proportion of variance.

\section{DISCUSSION}

Descriptively, the ten phonological tasks broke down into three groups. Three tasks that required a rhyming response were very easy. Ceiling effects were apparent on these three tasks. The distribution of responses was negatively skewed and the variance was highly restricted. As a result of the restriction of range these three tasks displayed low correlations with the other phonological tasks and with first-grade reading ability. One task (strip initial consonant) was extremely difficult. The distribution of responses on this variable was positively skewed and displayed a tendency toward bimodality. The other six measures were of intermediate difficulty and had distributions of responses that were more nearly symmetrical.

The seven nonrhyming tasks were highly interrelated. Despite the differing task requirements there was every indication that these tasks were tapping a similar construct. The mean correlation between the seven tasks was quite high, considering the reliability of the measures. The squared multiple correlations for each of these variables was also quite high. The visual impression that the correlations in Table 2 are uniformly moderate and do not tend to cluster was confirmed by the factor analysis, 
which extracted only one factor upon which each of the seven variables loaded highly. Regression analyses predicting first-grade reading ability confirmed the essential redundancy of these seven variables. Stepwise regression stopped after only two variables had entered the equation. The proportion of variance accounted for did not change appreciably when pairs of variables not chosen by the stepwise procedure were used as predictors. Although caution in interpreting multivariate statistics is always advised when the subjects to variables ratio is in the range employed in our investigation, the convergence of all of the analyses suggests that the data patterns are probably robust.

Performance on each of the seven nonrhyming tasks was related to first-grade reading ability. The absolute magnitude of the performance difference between skilled and less-skilled readers is apparent in Table 3 and in many cases is quite large, considering that the partitioning represents not an extreme groups comparison but instead is a median split. At the end of kindergarten the skilled readers were near ceiling on several tasks, while the less-skilled readers were getting barely half of the items correct.

The correlational data also suggest that the seven nonrhyming tasks are quite impressive predictors of first-grade reading ability. All of the correlations with Metropolitan scores were significant and at least of moderate strength. The magnitude of the correlations must be considered in the context of the reliability of the tasks (each administered in a very short time span) and in the context of the type of correlations that are obtained when more comprehensive and carefully standardized measures of cognitive skills arc employed. From this standpoint, the diagnosticity of the phonological measures was truly impressive. All seven nonrhyming measures correlated with first-grade reading more strongly than did a standardized IQ test (see Stanovich, Cunningham, \& Feeman, 1984, where a similar result obtained when all the tests were administered at the end of first grade). Three of the phonological tasks displayed correlations with first grade reading as large as those obtained from a standardized readiness test that was designed to tap a variety of reading-related cognitive skills. A stepwise regression of the Metropolitan scores on the phonological variables indicated that the latter explained $66.2 \%$ of the variance. When each possible pair of nonrhyming phonological measures served as predictors the median proportion of variance explained was $57.6 \%$. This compares to $59.1 \%$ explained by the Metropolitan readiness test and Otis-Lennon IQ in combination.

The results of this investigation bode well for the future use of phonological awareness measures in both research and educational settings. The wide variety of tasks that have been employed appear to be tapping a similar ability. Results from different investigations are probably not too contaminated by disparate task requirements. In fact, the degree of 
task convergence was quite a surprise to the present investigators who entered upon this investigation fully prepared to uncover the fact that the variance introduced by differing cognitive requirements would tend to obscure the underlying phonological abilities that were being tapped and would lead to vastly different patterns of task performance.

Finally, the uniformly moderate predictive accuracy of each task, coupled with the impressive predictive power when sets of these measures are used together, is an encouraging sign as regards future practical applications. While we must reiterate the caution that the relationship between reading ability and phonological awareness seems to be characterized by reciprocal causation (see Baron \& Treiman, 1980; Ehri, 1979; Morais, Cary, Alegria, \& Bertelson, 1979; Perfetti et al., 1981), the causal connection at the earliest stages of reading acquisition is probably most strong from phonological awareness to increased reading acquisition (Bradley \& Bryant, 1983; Treiman \& Baron, 1983).

\section{REFERENCES}

Backman, J. (1983). The role of psycholinguistic skills in reading acquisition: A look at early readers. Reading Research Quarterly, 18, 466-479.

Baron, J., \& Treiman, R. (1980). Use of orthography in reading and learning to read. In J. Kavanagh \& R. Venezky (Eds.), Orthography, reading, and dyslexia. Baltimore: Univ. Park Press.

Bradley, L., \& Bryant, P. (1978). Difficulties in auditory organization as a possible cause of reading backwardness. Nature (London), 271, 746-747.

Bradley, L., \& Bryant, P. (1983). Categorizing sounds and learning to read: A causal connection. Nature (London), 301, 419-421.

Bruce, D. (1964). The analysis of word sounds by young children. British Journal of Educational Psychology, 34, 158-170.

Calfee, R., Chapman, R. \& Venezky, R. (1972). How a child needs to think to learn to read. In L. Gregg (Ed.), Cognition in learning and memory. New York: Wiley.

Calfee, R., Lindamood, P., \& Lindamood, C. (1973). Acoustic-phonetic skills and reading: Kindergarten through twelfth grade. Journal of Educational Psychology, 64, 293-298.

Ehri, L. (1979). Linguistic insight: Threshold of reading acquisition. In T. Walker \& G. Mackinnon (Eds.), Reading research: Advances in research and theory (Vol.1). New York: Academic Press.

Fox, B., \& Routh, D. (1976). Phonemic analysis and synthesis as word attack skills. Journal of Educational Psychology, 68, 70-74.

Fox, B., \& Routh, D. (1980). Phoncmic analysis and severc reading disability. Journal of Psycholinguistic Research, 9, 115-119.

Fox, B., \& Routh, D. (1983). Reading disability, phonemic analysis, and dysphonic spelling: A follow-up study. Journal of Clinical Child Psychology, 12, 28-32.

Golinkoff, R. (1978). Phonemic awareness skills and reading achievement. In F. Murray \& J. Pikulski (Eds.), The acquisition of reading. Baltimore: Univ. Park Press.

Helfgott, J. (1976). Phonemic segmentation and blending skills of kindergarten children: Implication for beginning reading acquisition. Contemporary Educational Psychology, 1, 157-169.

Jorm, A., \& Share, D. (1983). Phonological recoding and reading acquisition. Applied Psycholinguistics, 4, 103-147.

Jusczyk, P. (1977). Rhymes and reasons: Some aspects of the child's appreciation of poetic form. Developmental Psychology, 13, 599-607. 
Lewkowicz, N. (1980). Phonemic awareness training: What to teach and how to teach it. Journal of Educational Psychology, 72, 686-700.

Liberman, I. (1973). Segmentation of the spoken word and reading acquisition. Bulletin of the Orton Society, 23, 65-77.

Liberman, I. (1982). A language-oriented view of reading and its disabilities. In H. Myklebust (Ed.), Progress in learning disabilities (Vol. 5). New York: Grune \& Stratton.

Marsh, G., \& Mineo, R. (1977). Training preschool children to recognize phonemes in words. Journal of Educational Psychology, 69, 748-753.

Morais, J., Cary, L., Alegria, J., \& Bertelson, P. (1979). Does awareness of speech as a sequence of phones arise spontaneously?. Cognition, 7, 323-331.

Perfetti, C., Beck, I., \& Hughes, C. (1981, March). Phonemic knowledge and learning to read. Paper presented at The meeting of the Society for Research in Child Development, Boston.

Rozin, P., \& Gleitman, L. (1977). The structure and acquisition of reading: II. The reading process and the acquisition of the alphabetic principle. In A. Reber \& D. Scarborough (Eds.), Toward a psychology of reading. Hillsdale, NJ: Erlbaum.

Stanovich, K., Cunningham, A., \& Feeman, D. (1984). Intelligence, cognitive skills, and early reading progress. Reading Research Quarterly, 19, 120-139.

Tabachnick, B., \& Fidell, L. (1983). Using multivariate statistics. New York: Harper \& Row.

Treiman, R., \& Baron, J. (1981). Segmental analysis ability: Development and relation to reading ability. In T. Waller \& G. Mackinnon (Eds.), Reading research: Advances in theory and practice (Vol. 3). New York: Academic Press.

Treiman, R., \& Baron, J. (1983). Phonemic-analysis training helps children benefit from spelling-sound rules. Memory \& Cognition, 11, 382-389.

Williams, J. (1980). Teaching decoding with an emphasis on phoneme analysis and phoneme blending. Journal of Educational Psychology, 72, 1-15.

ReCEIVED: October 17, 1983; REVISED: January 11, 1984. 